\title{
Nanoplatform Self-assembly From Small Molecules of Porphyrin Derivatives for NIR-II Fluorescence Imaging Guided Photothermal-immunotherapy
}

\section{Yanbing Cao}

Fuzhou University

De Wei

Fujian Provincial Hospital

\section{Lixia Yang}

Wuhan Institute of Technology

\section{Zijin Luo}

Wuhan Institute of Technology

\section{Peiwen Yu}

Mengchao Hepatobiliary Hospital of Fujian Medical University

Hao Li

Mengchao Hepatobiliary Hospital of Fujian Medical University

\section{Cao Zhang}

Mengchao Hepatobiliary Hospital of Fujian Medical University

Xiaolong Liu

Mengchao Hepatobiliary Hospital of Fujian Medical University https://orcid.org/0000-0002-3096-4981

Fengshou Wu ( $\square$ fswu@wit.edu.cn )

Wuhan Institute of Technology

\section{Ming Wu}

Wuhan Institute of Technology

\section{Yongyi Zeng}

Mengchao Hepatobiliary Hospital of Fujian Medical University

\section{Research}

Keywords: Porphyrin derivatives, Self-assembly, Photothermal conversion, NIR-II fluorescence imaging, PD-1 antibody, Photothermal-immunotherapy

Posted Date: September 2nd, 2021

DOI: https://doi.org/10.21203/rs.3.rs-855967/v1 
License: (c) (i) This work is licensed under a Creative Commons Attribution 4.0 International License. Read Full License

Version of Record: A version of this preprint was published at Advanced Healthcare Materials on February 8th, 2022. See the published version at https://doi.org/10.1002/adhm.202102526. 


\section{Abstract}

Combinatorial photothermal and immunotherapy have demonstrated great potential to remove primary tumors, suppress metastases, and prevent tumor recurrence. However, this strategy still confronts with many limitations, such as complex components, sophisticated construction and inadequate therapeutic efficacy. In this work, we develop small molecules of porphyrin derivatives (PPor) which can selfassemble into monodispersed nanoparticles without supplement of any other ingredients or surfactants. The formed PPor nanoparticles (PPor NPs) exhibit highly photothermal conversion efficiency of $70 \%$ and NIR-II luminous abilities originated from the strong intramolecular charge transfer (ICT) effect of D-A structure under $808 \mathrm{~nm}$ laser irradiation, thus achieving NIR-II fluorescence imaging guided photothermal therapy (PTT) against primary tumor with a high cure rate. More importantly, tumor-associated antigens (TAAs) together with damage-associated molecular patterns (DAMPs) released from PTT-treated cancer cells are proved to elicit immune responses in some degree. After combination with programmed cell death-1 (PD-1) antibody, a robust systematic antitumor immunity are generated to restrain both primary and abscopal tumors growth, prolong survival, prevent pulmonary metastasis on an aggressive 4T1 murine breast tumor model. Thus, this study provides a promising therapeutic paradigm with porphyrin derivatives nano-assembly as phototheranostic agents for the treatment of aggressive tumor with high efficiency.

\section{Introduction}

Cancer immunotherapy has exhibited tremendous potential as the next generation of highly effective therapy modality, due to its ability to harness or liberate the inherent immune systems of patient to attack cancer cells, which is very different from the traditional mainstream therapy, such as resection, chemotherapy, and radiotherapy that directly kill or remove cancer cells [1]. The major types of cancer immunotherapy include cytokine therapy [2], immune checkpoint-blockade therapy (ICB) [3], cancer vaccines [4], as well as adoptive cell transfer therapy [5]. Particularly, ICB using antibodies as the inhibitors to interdict immunosuppressive pathways, such as CTLA-4 and PD-1/PD-L1, has obtained remarkably clinical success in many different advanced cancers [6]. Nevertheless, only a small portion of patients whose tumor carry high neoantigen burden [7], sufficient tumor-infiltrating lymphocytes (TILs) [8], and PD-L1 expression [9] seem to receive durable clinical benefits from ICB. Although enhanced ICB efficacy could be achieved through combination with chemotherapy [10], radiotherapy [11], phototherapy [12], and other types of therapeutic modalities [13], how to safely and effectively elevate antitumor immune response is still challenging for substantial therapeutic benefits.

Photothermal therapy (PTT) has attracted intensive attention as a new paradigm for precise cancer therapy through photothermal conversion agents (PTCAs) to ablate tumor tissue through a noninvasive and spatiotemporal manner [14]. Recent studies have documented that PTT can elicit antitumor immune response via causing immunogenic cell death (ICD) of the cancer cells [15], along with the exposure of calreticulin (CRT) from endoplasmic reticulum to membrane, thereby facilitating the uptake by antigen presenting cells (APCs) [10]. Meanwhile, adenosine triphosphate (ATP) released from damaged tumor 
cells will accelerate intratumoral infiltration of APCs [16]. Furthermore, the high mobility group box-1 (HMGB-1) located in the nucleus will leak out to the extracellular matrix for providing adjuvant stimuli to APCs by binding with toll-like receptors [17]. However, the activity of tumor-infiltrated cytotoxic T lymphocytes (CTLs) is suppressed by the immune checkpoint signaling of PD-1/PD-L1 between CTLs and tumor cells [18]. Thus, combining the PD-1/PD-L1 checkpoint blockade with PTT could stimulate robust antitumor immunities to inhibit both primary and metastasis tumors. Up to now, numerous inorganic materials (carbon nanotubes [19], 2D nanosheet [20], gold nanoparticles [21], CuS nanoparticles [22], prussian blue nanoparticle [23], etc) and organic materials (PLGA-ICG-R837 [15],

NLG919/IR780/PEG-PCL [24], R848/polydopamine [25], semiconducting polymer [26], etc) have been developed as PTCAs for photothermal-immunotherapy. However, it still exists many limitations by using this strategy, including complex components, sophisticated preparation, undefined structure, lack of diagnostic function or insufficient therapeutic efficacy. In this context, versatile theranostic agents with molecular imaging and PTT effect in single molecular structure are highly pursued for actual applications [27].

Porphyrin-based organic molecule, a key component of hemoglobin with light-absorbing ability, is characterized with good biocompatibility, red absorption and fluorescence imaging in first near-infrared (NIR-I) region [28]. Nevertheless, owing to the limited water solubility, most of them are need to blend with other emulsifying agents and solubilizers in clinical applications, accounting for a major weight proportion that restricts the encapsulating capacity and leads to undesired side effect[29]. Furthermore, compared with NIR-I, the second near-infrared region (NIR-II) has the advantages of deeper tissue penetration and little auto-fluorescence and thus shows superior imaging behavior than NIR-I [27]. Introducing donor-acceptor (D-A) architecture into fluorophores is an effective strategy for shift the fluorescent emission into NIR-II window, but also accompanied with the decrease of emission efficiency due to the excited state more likely gets back to the ground state by the nonradiative decay pathways [30]. From another perspective, the nonradiative decay of excited state is the main energy source for PTT. Under these circumstances, a single porphyrin-derived fluorophore simultaneously bearing NIR fluorescence and photothermal conversion capacity needs to rational molecular design to balance radiative and nonradiative pathways of excited state. Our groups and others have developed various amphiphilic porphyrins with simple molecular structure which can self-assembly into nanoparticles for PTT treatment [28, 29, 31-34], but most of them have no fluorescence emission due to the aggregation caused quenching (ACQ) effect and the narrowed optical gap, not to mention bearing NIR-II emission.

Herein, we developed a new amphiphilic porphyrin (PPor) derivate conjugating with two poly(ethylene glycol) (PEG) chains to improve the water solubility of the porphyrin. Meanwhile, perylene diimide, as a strong electron acceptor, was also integrated into the porphyrin skeleton to form D-A structure, thus would decrease the energy bandgap and subsequently redshift the absorption. PPor molecules can selfassemble into monodispersed nanoparticles (PPor NPs) with regular spherical shape, which not only act as a PTT therapeutic agent, but also provide NIR-II fluorescence signal with quantum yield of $0.4 \%$, comparable to other small-molecule dyes with D-A structure [35]. PPor NPs were demonstrated to show enhanced tumor retention than other small molecular fluorophores like ICG (a FDA approved fluorescence 
contrast and PTT agent), which is suitable for local injection to overcome stromal barrier of solid tumors. In addition, PPor NPs exhibited photostability and good colloidal dispersion in physiological conditions, guaranteeing a reliable drug dose and a steady laser irradiation condition after localized injection [36]. Through only one localized injection and one irradiation treatment, PPor NPs could completely ablate primary tumor. More importantly, the PTT-treated tumors could release adequate DAMPs (e.g., CRT and HMGB-1) and tumor-associated antigens, which elicited robust systemic antitumor immunity after incorporation with PD-1 antibody to attack distant tumor cells. Such a strategy also evoked the strong immune-memory effect to inhibit tumor metastasis (Fig. 1). Thus, this work presented an effective NIR-II fluorescence imaging guided photothermal-immunotherapy for eradicating invasive cancer cells by using a simple formulation of self-assembled porphyrin derivatives.

\section{Experimental Section}

\section{Synthesis of PPor and PPor NPs}

The synthesis methods of PPor are presented in Additional file 1. For self-assembly of PPor into PPor NPs, $5 \mathrm{~mL}$ deionized water was added.into a solution of PPor in tetrahydrofuran $(100 \mu \mathrm{L}, 1 \mathrm{mg} / \mathrm{mL})$. After stirring at room temperature for half an hour, the organic solvent was removed by air blowing. The mixture was purified by centrifugation to remove agglomerated particles, yielding the clear aqueous solution of PPor NPs with dark brown color. The characterization of PPor and PPor NPs was clarified in Additional file 1

\section{Photothermal performance of PPor NPs}

PPor NPs at different concentrations in water were respectively exposed under $808 \mathrm{~nm}$ laser $\left(1.5 \mathrm{~W} / \mathrm{cm}^{2}\right.$, $600 \mathrm{~s}$ ). Meanwhile, for investigating the influence of laser power on photothermal behavior, PPor NPs aqueous solutions $(20 \mu \mathrm{g} / \mathrm{mL})$ was irradiated with different power density $\left(0.3,0.6,0.9,1.2,1.5 \mathrm{~W} / \mathrm{cm}^{2}\right)$. To further explore the photothermal stability, $1 \mathrm{~mL}$ of PPor NPs was exposed to laser irradiation (808 nm, $1.5 \mathrm{~W} / \mathrm{cm}^{2}$ ) for $10 \mathrm{~min}$ and laser was temporarily turned off until the temperature drops to the room temperature. This procedure was repeated 5 times.

\section{In vitro photothermal therapy}

To explore photothermal therapy effect of PPor NPs, 4T1 cells cultured in a 96 -well plate (10000 cells per well) were incubated with PPor NPs at various concentrations $(0,5,8,10,12,14$, and $16 \mu \mathrm{g} / \mathrm{mL})$. With further $8 \mathrm{~h}$ of incubation, the cells received $808 \mathrm{~nm}$ laser irradiation $\left(0.8 \mathrm{~W} / \mathrm{cm}^{2}, 10 \mathrm{~min}\right)$. After further 24 $\mathrm{h}$ of incubation, the cell viability was measured by CCK-8 assay.

Live/dead cell assay and apoptosis assay were also used to determine the PTT efficacy of PPor NPs. For live/dead cell assay, 4T1 cells underwent the above treatments were labeled by calcein AM and PI, which then imaged by a fuorescence microscope (Zeiss Axio Vert. A1, Germany). With respect to apoptosis 
assay, the cells were labeled by Annexin V-FITC/PI solution, and then analyzed by flow cytometry (BD, FACSVerse, USA).

\section{CRT expression after PTT}

4T1 cells in the confocal dishes received the above treatments were incubated with the primary antibody of CRT for $2 \mathrm{~h}$ and secondary antibody for $1 \mathrm{~h}$. Then, the cells were stained with Hoechst for 10 min and observed by CLSM (Zeiss LSM780).

\section{In Vitro DC maturation assay}

BMDC cells were isolated from bone marrow according to our previously reported method [13]. Then, the supernatant of 4T1 cells underwent aforementioned PTT treatments was added into BMDCs for triggering their maturation. After $48 \mathrm{~h}$ of incubation, BMDC cells were collected, and co-stained with CD11c-APC (eBioscience $\left.{ }^{\mathrm{TM}}, 17-0114-82\right)$, CD80-PE (eBioscience ${ }^{\mathrm{TM}}$, 12-0801-82) and CD86-PE-Cy7 (eBioscience $\left.^{\mathrm{TM}}, 25-0862-82\right)$. Finally, the cells were analyzed by a flow cytometer (BD, FACSVerse, USA).

\section{In vivo NIR-II fluorescence imaging}

To establish tumor model, $4 \mathrm{~T} 1$ cells $\left(5 \times 10^{5}\right)$ were subcutaneously injected into the mice flank. When volume of tumor grew about $150 \mathrm{~mm}^{3}$, PPor NPs were locally injected into the tumor $(50 \mu \mathrm{L}, 0.4 \mathrm{mg} / \mathrm{mL})$, free ICG was used as the control. At different predetermined time points, the mice were anesthetized and scanned by an in vivo NIR II fluorescence imaging system (UNITED WELL).

\section{In vivo photothermal therapy}

BALB/c mice implanted with 4T1 tumor were randomly allocated to 4 groups with different treatments:

(1) Intratumoral injection of PBS without 808 nm laser;

(2) Intratumoral injection of PBS with $808 \mathrm{~nm}$ laser $\left(0.8 \mathrm{~W} / \mathrm{cm}^{2}, 10 \mathrm{~min}\right)$;

(3) Intratumoral injection of PPor NPs without $808 \mathrm{~nm}$ laser;

(4) Intratumoral injection of PPor NPs with $808 \mathrm{~nm}$ laser $\left(0.8 \mathrm{~W} / \mathrm{cm}^{2}, 10 \mathrm{~min}\right)$;

During the laser irradiation, the photothermal performance of PPor NPs was recorded by an IR thermal camera (Ti25 Fluke Co, USA), and the tumor growth was carefully monitored

To explore the histological changes in the tumors, one mouse in each group was euthanized after $24 \mathrm{~h}$ of PTT treatment. The tumor was collected, fixed in $4 \%$ formalin solution, dehydrated, deparaffinization and subjected to H\&E staining, Ki67 immunohistochemical and other immunofluorescence staining analysis.

\section{Antitumor evaluation on bilateral tumor}


To establish a bilateral tumor model, $1 \times 10^{6}$ cells and $0.5 \times 10^{6}$ of $4 \mathrm{~T} 1$ cells were respectively injected into the right and left flank of mice as primary tumor and distant tumor. Seven days later, the mice were randomly allocated to 5 groups receiving following treatments:

(1) Intratumoral injection of PBS for primary tumor;

(2) Intratumoral injection of PPor NPs for primary tumor;

(3) Intratumoral injection of PPor NPs with laser irradiation for primary tumor $\left(0.8 \mathrm{~W} / \mathrm{cm}^{2}, 10 \mathrm{~min}\right)$;

(4) Intravenous injection of aPD-1 (2.5 mg/kg);

(5) Intratumoral injection of PPor NPs with laser irradiation for primary tumor ( $\left.0.8 \mathrm{~W} / \mathrm{cm}^{2}, 10 \mathrm{~min}\right)$. After $24 \mathrm{~h}$, aPD-1 was intravenously injected into mice $(2.5 \mathrm{mg} / \mathrm{kg})$;

The therapeutic effect on both primary and abscopal tumor were analyzed according to the aforementioned method. Mice were sacrificed when exhibited signs of impaired health or when the tumor volume exceeded $1200 \mathrm{~cm}^{3}$. Treatment-induced CRT expression and HMGB-1 release and T lymphocyte infiltration were checked by immunofluorescence and immunohistochemical analysis, respectively. To analysis treatment-induced cytokine secretion, the abscopal tumors were ground and homogenized, and then, the supernatant was collected to detect cytokines (IFN- $\gamma$, Boster, EK0375; Granzyme B, Boster, EK1115).

\section{Immune memory effect}

The 4T1 tumor-bearing mice underwent the aforementioned treatments. At the 20 days, the tumor surgically was removed. Seven days later, $0.5 \times 10^{6} 4 \mathrm{~T} 1$ cells were implanted into the mice through caudal vein. After 2 weeks, the lungs were isolated from treated mice to photograph, and further fixed in $4 \%$ formalin solution for H\&E staining.

\section{Statistical analysis}

The experimental data were presented with the mean \pm standard deviation. Significance among different groups was calculated using One-way ANOVE (and nonparametric) with post-hoc tests. Survival rate in different groups was compared using a Log-Rank test. All statistical analyses were carried out by GraphPad Prism 6.01. The P-value $<0.05$ was considered as statistically significant.

\section{Results And Discussion}

\section{Fabrication and characterization of PPor and PPor NPs}

PPor was synthesized according to our previously reported methods with slight modification [31]. As shown in Fig. S1, triethylene glycol monoethyl ether was grafted in the 10- and 20-meso positions of porphyrin to increase its water solubility, and 5- and 15-meso positions were conjugated with the electron 
acceptor of perylene diimide through $\pi$-conjugated triple bond. The structure of the as-prepared PPor was proved by ${ }^{1} \mathrm{H}$ NMR (Fig. 2a and Fig. S2), ${ }^{13} \mathrm{C}$ NMR (Fig. S3) and mass spectrum (Fig. S4). Due to the presence of hydrophobic and hydrophilic groups, PPor can spontaneously self-assemble into nanostructure (named as PPor NPs) though $\pi-\pi$ stacking and hydrophobic interaction, verified by the transmission electron microscopy (TEM) which showed a spherical-like shape with the average diameter of 50-100 nm. This result is consistent with the average hydrodynamic size determined by dynamic light scattering (DLS) (Fig. 2b and Fig. S5). The zeta potential of PPor NPs was measured to $-32.2 \mathrm{mV}$ (Fig. 2c), ensuring their highly colloid stability in physiological condition without protein absorption (Fig. S6).

The optical properties of Por and PPor NPs were investigated by testing their absorption and fluorescence spectra. As shown in Fig. 2d, PPor displayed an obvious NIR absorbance centered at $860 \mathrm{~nm}$, ascribing to the intramolecular charge transfer (ICT) from the electron donor to electron acceptor. As expected, HOMO orbital was predominantly positioned in the porphyrin part while the LUMO orbital was in perylene diimide part, with a narrow energy bandgap of $0.6548 \mathrm{eV}$ (Fig. S7). After self-assembled into PPor NPs, the spectrum showed a distinctively red-shifted and broadened pattern, probably due to $\pi-\pi$ stacking of the PPor molecules in nanostructure [31]. Meanwhile, PPor in organic solution exhibited obvious NIR-II emissions from 850 to $1200 \mathrm{~nm}$, with the fluorescence quantum yield $\left(\Phi_{F}\right)$ of $1.37 \%$, higher than most of the reported small molecules [37]. PPor NPs in water showed red-shifted emission pattern with maximum emission wavelength at $1000 \mathrm{~nm}$ (Fig. 2e). The $\Phi_{F}$ of PPor NPs was calculated to be $0.4 \%$ (Fig. S8), which was comparable with other NIR-II emissive nanoparticles [38, 39].

\section{Photothermal performance of PPor NPs}

Considering the strong NIR-I absorption of PPor NPs, their photothermal conversion abilities were systematically studied. The temperature elevation of PPor NPs was found to depend on the laser power intensity, irradiation time, as well as NPs' concentration (Fig. 2f, g). In particular, the temperature of the aqueous solution of PPor NPs dramatically reached above $55^{\circ} \mathrm{C}$ under $808 \mathrm{~nm}$ laser irradiation (1.5 $\mathrm{W} / \mathrm{cm}^{2}, 10 \mathrm{~min}$ ), compared with pure water with negligible temperature elevation at the same condition. The photothermal conversion efficiency (PCE) was equal to 70\% (Fig. 2h and Fig. S9), higher than the values of most reported PTCAs [40]. In addition, PPor NPs possess outstanding photothermal stability, with similar performance even underwent five times of heating/cooling processes (Fig. 2i). Accordingly, the absorbance of PPor NPs did not change with 10 min of continuous laser irradiation (Fig. S10). These results indicated that as-prepared PPor NPs exhibit great efficacy for long-term PTT with repeated laser irradiation.

In vitro photothermal therapy

Before exploration of the photothermal ablation of cancer cells by PPor NPs, we first assessed their in vitro cytotoxicity in different cell types including normal cells (NIH 3T3) and tumor cells (4T1). The results showed that PPor NPs without laser irradiation had negligible cytotoxic effect in the concentration ranged 
from 0 to $25 \mu \mathrm{g} / \mathrm{mL}$ (Fig. 3a), demonstrating that the PPor NPs hold good biocompatibility for subsequent applications. Next, to prove the photothermal therapeutic effect caused by PPor NPs, the 4T1 cells treated with PPor NPs were exposed by $808 \mathrm{~nm}$ laser irradiation $\left(0.8 \mathrm{~W} / \mathrm{cm}^{2}, 10 \mathrm{~min}\right)$. As shown in Fig. 3b, upon laser irradiation, PPor NPs displayed a dose-dependent cytotoxicity against 4T1 cancer cells, with a cell viability decrease to $30 \%$ at $10 \mu \mathrm{g} / \mathrm{mL}$. To further confirm the PTT effect of PPor NPs, the treated cells were stained with calcein $\mathrm{AM}$ and propidium iodide $(\mathrm{PI})$ to intuitively observe their survival states (Fig. 3c). Similar with control group, the cells showed an entire green fluorescence without cytotoxicity when only irradiated with laser or only incubated with PPor NPs, revealing no obvious phototoxicity from laser or dark cytotoxicity from PPor NPs alone. However, with the combination of laser irradiation and PPor NPs incubation, the cells were mostly killed. Moreover, the PTT effect of PPor NPs was also checked by Annexin V-FITC/PI assay (Fig. 3d). Most significantly, PPor NPs supplemented with laser irradiation showed the highest ratio of cell apoptosis (95.93\%). These results are consistent with the above CCK-8 assay, suggesting the great potential of PPor NPs for cancer PTT treatment.

\section{PTT induced immunogenic cell death (ICD)}

Recently, PTT has been reported to generate antitumor immunological effects to some extent by inducing immunogenic cell death (ICD) to expose DAMPs and TAAs. Calreticulin (CRT) is considered as one of the representative DAMPs, and we selected it as the indicator to confirm whether PTT effect of PPor NPs can induce ICD. From CLSM results in Fig. 4a, PPor NPs supplemented with laser irradiation (PPor NPs + Laser) treatment induced intensive CRT exposure on $4 \mathrm{~T} 1$ cells, while hardly observed from the cells treated only with laser irradiation or PPor NPs.

We further evaluated the effects of DMAPs and TAAs released from PTT-induced dead cells on the maturation of DCs for initiating the immune responses, according to following experiments (Fig. 4b). The supernatant of $4 \mathrm{~T} 1$ cells underwent the aforementioned treatments was incubated with fresh immature DCs derived from bone marrow cells (BMDCs), and the BMDC maturation was analyzed by using FACS to detect the expressions of the co-stimulatory molecules of CD80 and CD86 [13]. The result demonstrated that the expression level of CD80 and CD86 on BMDCs in the treatment group of PPor NPs with laser irradiation was much higher than other groups which could not efficiently induce ICD of cancer cells (Fig. 4c). Taken together, these results suggest that PPor NPs can not only ablate cancer cells, but also induce ICD response to elicit antitumor immunity by PTT effect under external laser irradiation.

\section{In vivo NIR-II fluorescence imaging and photothermal therapy}

Because of colloid- and photo- stability, PPor NPs might be used for NIR-II fluorescence imaging and PTT. To investigate these properties in vivo, a subcutaneous $4 \mathrm{~T} 1$ breast tumor model was established. The therapeutic schedule was depicted in Fig. 5a. Importantly, localized injection was employed in our experiment, considering the dense tumor stroma to inhibit the drug accumulation and penetration after systemic administration. Before therapeutic assessment, we investigated the in vivo distribution and tumor retention of PPor NPs, while ICG (a FDA-approved dye with NIR I/II fluorescence and PTT 
performance) was taken as the control. NIR II fluorescence signal in PPor NPs group mainly located in tumor tissue for more than $48 \mathrm{~h}$, while free ICG easily leaked out and cleared by liver even at $8 \mathrm{~h}$ postinjection (Fig. 5b), highlighting the advantages of stromal hindrance that precludes nanoscale-sized NPs circulation out from tumor tissues.

Next, the in vivo PTT effect of PPor NPs was assessed. Mice were randomly allocated to four groups (localized injection of PBS or PPor NPs, and then with or without laser irradiation). As recorded by IR thermal camera (Fig. 5c, d), PPor NPs displayed an obvious photothermal conversion behavior in tumor upon laser irradiation. Afterwards, PTT therapeutic effect was investigated by monitoring tumor size during the treatment process. The tumors with combined treatment of PPor NPs and laser irradiation (PPor NPs + Laser) were effectively ablated with rather slow growth, while the tumor volumes in PBS, PBS + Laser, and PPor NPs groups grew rapidly similar to that of PBS group (Fig. 5e), demonstrating the remarkable PTT antitumor efficacy of PPor NPs as the average temperature of the tumors in this group increased to $55 \pm 5.76^{\circ} \mathrm{C}$, which is high enough to ablate tumor. The tumor weights and photographs at the endpoint also supported that PTT of PPor NPs was responsible for tumor growth repression (Fig. $5 f, g$ and Fig. S11). Besides, histological examination of the treated tumor (H\&E, Ki67 as well as TUNEL) was utilized to confirm antitumor effect of PTT. As a result, PPor NPs + Laser group displayed considerably enhanced cell damage when compared with other groups (Fig. 5h and Fig. S12). Furthermore, immunofluorescence staining of the tumor sections for CRT and HMGB-1 was used to identify whether PTT effect could induce ICD of cancer cells in vivo. As shown in Fig. 5h, PPor NPs + Laser induced significant CRT expression on the cell surface. HMGB-1 is another danger signal to stimulate phagocytosis of the dead cells by APCs including DCs. As anticipated, HMGB-1 was dramatically released from the nucleus in PPor NPs + Laser group, compared with other groups in which the HMGB-1 predominantly located in the nucleus. Collectively, the CRT exposure and HMGB-1 release cumulatively verified PPor NPs-based PTT could significantly induce ICD in vivo. Meanwhile, the localized administration of PPor NPs was well-tolerated in animal safety studies, without evidence of obvious body weight fluctuation or accidental death (Fig. S13).

\section{In vivo combination effect of PTT and ICB}

Apart from direct tumor ablation as a local treatment modality, PTT can induce ICD to trigger antitumor immunity to some degree, but the immune response (abscopal effect) is relatively too weak and shortlived to suppress the growth of remaining or spread tumor nodes [41]. Combination of PTT with immune therapeutic approaches has emerged as a glamorous method for boosting cancer treatment effect [42]. In this situation, we further explored whether the reinforced immune responses arising from PTT and immunotherapy combination were strong enough to suppress the untreated distant tumor. To this end, a bilateral tumor model was constructed by inoculating $1 \times 10^{6}$ and $0.5 \times 10^{6}$ tumor cells (4T1) into the right and left flanks of each BABL/c mouse, respectively (Fig. 6a). The right tumors were designed for localized PTT treatment, while the left tumors without direct treatment were taken as an artificial distant tumor model. Until the primary tumors reached about $100 \mathrm{~mm}^{3}$, mice received the following treatments ( $n=7$ per group): (1) PBS; (2) PPor NPs alone; (3) PPor NPs + Laser; (4) aPD-1; (5) PPor NPs + Laser + 
aPD-1. PBS or PPor NPs was intratumorally injected to right tumor of each mouse. One day after injection, the tumors with NPs in group 3 and 5 were exposed to the $808 \mathrm{~nm}$ laser $\left(0.8 \mathrm{~W} / \mathrm{cm}^{2}\right)$ for $10 \mathrm{~min}$. After one day of PTT treatment, aPD-1 was intravenously (i.v.) injected into mice for group 4 and 5 (Fig. 6a). The antitumor effects was evaluated by observing the growth rates of both right and left tumors, respectively. As shown in Fig. 6b-e, compared with PPor NPs + Laser had only inhibition effect against primary tumor, PPor NPs + Laser + aPD-1 could simultaneously suppress primary and abscopal tumor with prolonged survival time, suggesting a remarkable systemic therapeutic outcome resulting from the promoted antitumor immune response evoked by the PTT and ICB combination effect. The body weights of mice were not found to loss in all groups, suggesting no systemic toxicity (Fig. S14).

To identify the antitumor immune response based on the combined PTT-immunotherapy with PPor NPs, the lymphocytes and the pro-inflammatory cytokines like IFN-y and Granzyme B in abscopal tumors were analyzed. From the multispectral immunofluorescent images of tumor slice (Fig. 6f), we found the frequency of $\mathrm{CD}^{+} \mathrm{T}$ cells in combination therapy (group 5), also named cytotoxic $\mathrm{T}$ lymphocytes (CTLs) which can potently recognize and kill tumor cells, was much higher than other groups. Meanwhile, as activated CTLs would secret IFNy, Granzyme B, and so on to stimulate cell apoptosis pathway to kill the tumor cells, we next determined the secretion of IFNY and Granzyme B in tumor tissue by ELISA. Consequently, the combination therapy induced highest level of IFNY and Granzyme B among all groups (Fig. 6g, h). Therefore, these results demonstrate that combined PTT-immunotherapy has the potential to completely eradicate the primary tumors, as well as significantly suppress abscopal tumors by eliciting robust systemic antitumor immunity.

Immune-memory effect is a distinct feature of adaptive immune responses to prevent tumor recurrences and metastases. To identify whether the combined PTT-immunotherapy could generate immune-memory effect, the mice bearing $4 \mathrm{~T} 1$ tumor firstly received the aforementioned treatments, followed by i.v.

injection of $4 \mathrm{~T} 1$ cells $\left(1 \times 10^{6}\right.$ cells per mouse) at day 27 to mimic the cancer cell spreading (Fig. $\left.7 \mathrm{a}\right)$. As depicted in the photographs and H\&E staining images of excised lung, the groups treated with PBS, PPor NPs alone, PPor NPs + Laser and aPD-1 all displayed macroscopical lung metastasis, but the combination of PPor NPs $+\mathrm{L}$ and aPD-1 completely restrained lung colonization of invasive 4T1 cells, which could confirm the excellent immune memory and protective effects induced by PPor NPs-mediated PTT and PD1 blockade (Fig. 7b, c).

\section{Conclusion}

In summary, we proposed a new small-molecule dye (PPor) based on porphyrin derivatives with excellent NIR-I absorption and NIR-II fluorescence emission for image-guided photothermal-immunotherapy. PPor could spontaneously self-assemble into nanoparticles (PPor NPs) without supplement of any extra ingredients and cargos because of its amphiphilic property. PPor NPs possessed regular diameter, high colloid- and photo- stability, and relatively high NIR-II fluorescence quantum yield (0.4\%) and NIR-I photothermal conversion efficiency $(70 \%)$ originating from the strong ICT effect of D-A structure. These impressive efficiency facilitates the application of PPor NPs in NIR-II fluorescence imaging-guided 
photothermal treatment. After injected within tumor sites, the PTT effect not only ablated the primary tumor entity in situ, but also induced ICD which could enhance systemic cancer immunotherapy of PD-1 antibody to inhibit distant tumor though improving infiltration of $\mathrm{CD} 8^{+}$cytotoxic $\mathrm{T}$ cells. Furthermore, this combination treatment also evoked strong immune-memory effect to attack and kill spreading metastatic tumors, as demonstrated on an artificial whole-body metastasis tumor model. Thus, these complementary theranostic effects of PPor NPs with explicit structure is particularly effective for refractory tumors characterized as limited drug penetration and are prone to recurrence/metastasis, which may advance toward clinical translation.

\section{Declarations}

\section{Ethics approval and consent to participate}

All animal experiments were carried out in accordance with national animal management regulations of China and approved by the Animal Ethics Committee of Mengchao Hepatobiliary Hospital of Fujian Medical University.

\section{Consent for publication}

All authors of this study agreed to publish.

\section{Availability of data and materials}

All data generated or analysed during this study are included in this published article (and its supplementary information files).

\section{Competing interests}

The authors declare that they have no competing interest.

\section{Funding}

This work was supported by the National Natural Science Foundation of China (grant no. 61727823 and 21601142), the Joint Research Projects of Health and Education Commission of Fujian Province (grant no. 2019-WJ-20), the Natural Science Foundation of Fujian Province of China (2020J02010), the Joint Funds for the Innovation of Science and Technology, Fujian Province (grant no. 2019Y9046), and the Scientific Foundation of Fuzhou Municipal Health Commission (grant nos. 2020-S-wt7 and 2020-S-wp6).

\section{Authors' contributions}

YC conducted optical characterization and cell experiments. DW performed animal experiments and wrote initial draft. LY prepared all the compounds and nanoparticles involved in this work. ZL, PY, HL and $\mathrm{CZ}$ participated in part of some experiments and data analysis. XL reviewed the manuscript. FW, MW and 
YZ conceived of the project, designed the work and supervised the study. All authors read and approved the final manuscript.

\section{Acknowledgements}

Not applicable.

\section{References}

1. Couzin-Frankel J. Cancer Immunotherapy. Science. 2013;342:1432-1433.

2. Momin N, Mehta NK, Bennett NR, Ma L, Palmeri JR, Chinn MM, Lutz EA, Kang B, Irvine DJ, Spranger $\mathrm{S}$, at al. Anchoring of intratumorally administered cytokines to collagen safely potentiates systemic cancer immunotherapy. Sci Transl Med. 2019;11:eaaw2614.

3. Iwai $\mathrm{Y}$, Ishida M, Tanaka Y, Okazaki T, Honjo T, Minato N, Involvement of PD-L1 on tumor cells in the escape from host immune system and tumor immunotherapy by PD-L1 blockade. Proc Natl Acad Sci. 2002;99:12293-12297.

4. Zhang H, You X, Wang X, Cui L, Wang Z, Xu F, Li M, Yang Z, Liu J, Huang P, et al. Delivery of mRNA vaccine with a lipid-like material potentiates antitumor efficacy through Toll-like receptor 4 signaling. Proc Natl Acad Sci. 2021;118:e2005191118.

5. Yang S, Wen J, Li H, Xu L, Liu Y, Zhao N, Zeng Z, Qi J, Jiang W, Han W, et al. Aptamer-engineered natural killer cells for cell-specific adaptive immunotherapy. Small. 2019; 15:1900903.

6. Wang C, Wang J, Zhang X, Yu S, Wen D, Hu Q, Ye Y, Bomba H, Hu X, Liu Z, et al. In situ formed reactive oxygen species-responsive scaffold with gemcitabine and checkpoint inhibitor for combination therapy. Sci Transl Med. 2018;10:eaan3682.

7. Schumacher TN, Schreiber RD. Neoantigens in cancer immunotherapy. Science. 2015;348:69-74.

8. Tang H, Wang Y, Chlewicki L, Zhang Y, Guo J, Liang W, Wang J, Wang X, Fu YX. Facilitating T cell infiltration in tumor microenvironment overcomes resistance to PD-L1 blockade. Cancer Cell. 2016;29:285-296.

9. Topalian SL, Taube JM, Anders RA, Pardoll DM. Mechanism-driven biomarkers to guide immune checkpoint blockade in cancer therapy. Nat Rev Cancer. 2016;16:275.

10. Lu J, Liu X, Liao Y-P, Salazar F, Sun B, Jiang W, Chang CH, Jiang J, Wang X, Wu AM, et al. Nanoenabled pancreas cancer immunotherapy using immunogenic cell death and reversing immunosuppression. Nat Commun. 2017;8:1811. 
11. Chao Y, Xu L, Liang C, Feng L, Xu J, Dong Z, Tian L, Yi X, Yang K, Liu Z. Combined local immunostimulatory radioisotope therapy and systemic immune checkpoint blockade imparts potent antitumour responses. Nat Biomed Eng. 2018;2:611-621.

12. Wang M, Song J, Zhou F, Hoover AR, Murray C, Zhou B, Wang L, Qu J, Chen WR. NIR-triggered phototherapy and immunotherapy via an antigen-capturing nanoplatform for metastatic cancer treatment. Adv Sci. 2019;6:1802157.

13. Wu M, Zheng D, Zhang D, Yu P, Peng L, Chen F, Lin Z, Cai Z, Li J, Wei Z, et al. Converting immune cold into hot by biosynthetic functional vesicles to boost systematic antitumor immunity. iScience. 2020;23:101341.

14. Zheng D, Yu P, Wei Z, Zhong C, Wu M, Liu X. RBC membrane camouflaged semiconducting polymer nanoparticles for near-infrared photoacoustic imaging and photothermal therapy. Nano-Micro Letters. 2020;12:1-17.

15. Chen Q, Xu L, Liang C, Wang C, Peng R, Liu Z. Photothermal therapy with immune-adjuvant nanoparticles together with checkpoint blockade for effective cancer immunotherapy. Nat Commun. 2016;7:13193.

16. Zhu Q, Sun F, Li T, Zhou M, Ye J, Ji A, Wang H, Ding C, Chen H, Xu Z, et al. Engineering oxaliplatin prodrug nanoparticles for second near-infrared fluorescence imaging-guided immunotherapy of colorectal cancer. Small. 2021;17:2007882.

17. Chang $M$, Wang $M$, Wang $M$, Shu M, Ding B, Li C, Pang M, Cui S, Hou Z, Lin J. A multifunctional cascade bioreactor based on hollow-structured $\mathrm{Cu}_{2} \mathrm{MoS}_{4}$ for synergetic cancer chemo-dynamic therapy/starvation therapy/phototherapy/immunotherapy with remarkably enhanced efficacy. Adv Mater. 2019;31:1905271.

18. Topalian SL, Drake CG, Pardoll DM. Immune checkpoint blockade: a common denominator approach to cancer therapy. Cancer cell. 2015;27:450-461.

19. Wang C, Xu L, Liang C, Xiang J, Peng R, Liu Z. Immunological responses triggered by photothermal therapy with carbon nanotubes in combination with anti-CTLA-4 therapy to inhibit cancer metastasis. Adv Mater. 2014;26:8154-8162.

20. He L, Nie T, Xia X, Liu T, Huang Y, Wang X, Chen T. Designing bioinspired 2D MoSe ${ }_{2}$ nanosheet for efficient photothermal-triggered cancer immunotherapy with reprogramming tumor-associated macrophages. Adv Funct Mater. 2019;29:1901240.

21. Ong C, Cha BG, Kim J. Mesoporous silica nanoparticles doped with gold nanoparticles for combined cancer immunotherapy and photothermal therapy. ACS Appl Bio Mater. 2019; 2:3630-3638. 
22. Lin X, Wang X, Li J, Cai L, Liao F, Wu M, Zheng D, Zeng Y, Zhang Z, Liu X. Localized NIR-II photoimmunotherapy through the combination of photothermal ablation and in situ generated interleukin-12 cytokine for efficiently eliminating primary and abscopal tumors. Nanoscale. 2021;13:1745-1758.

23. Cano-Mejia J, Burga RA, Sweeney EE, Fisher JP, Bollard CM, Sandler AD, Cruz CRY, Fernandes R. Prussian blue nanoparticle-based photothermal therapy combined with checkpoint inhibition for photothermal immunotherapy of neuroblastoma. Nanotechnol Biol Med. 2017;13:771-781.

24. Peng J, Xiao Y, Li W, Yang Q, Tan L, Jia Y, Qu Y, Qian Z. Photosensitizer micelles together with IDO inhibitor enhance cancer photothermal therapy and immunotherapy. Adv Sci. 2018;5:1700891.

25. Lu Q, Qi S, Li P, Yang L, Yang S, Wang Y, Cheng Y, Song Y, Wang S, Tan F. Photothermally activatable PDA immune nanomedicine combined with PD-L1 checkpoint blockade for antimetastatic cancer photoimmunotherapy. J Mater Chem B. 2019;7:2499-2511.

26. Li J, Yu X, Jiang Y, He S, Zhang Y, Luo Y, Pu K. Second near-infrared photothermal semiconducting polymer nanoadjuvant for enhanced cancer immunotherapy. Adv Mater. 2021;33:2003458.

27. Jiang R, Dai J, Dong X, Wang Q, Meng Z, Guo J, Yu Y, Wang S, Xia F, Zhao Z, et al. Improving imageguided surgical and immunological tumor treatment efficacy by photothermal and photodynamic therapies based on a multifunctional NIR AIEgen. Adv. Mater. 2021;33:2101158.

28. Yang M, Cao S, Sun X, Su H, Li H, Liu G, Luo X, Wu F. Self-assembled naphthalimide conjugated porphyrin nanomaterials with D-A structure for PDT/PTT synergistic therapy. Bioconjugate Chem. 2020;31:663-672.

29. Hou W, Lou JWH, Bu J, Chang E, Ding L, Valic M, Jeon HH, Charron DM, Coolens C, Cui D, et al. A nanoemulsion with a porphyrin shell for cancer theranostics. Angew Chem Int Ed. 2019;58:14974-14978.

30. Xi D, Xiao M, Cao J, Zhao L, Xu N, Long S, Fan J, Shao K, Sun W, Yan X, et al. NIR light-driving barrierfree group rotation in nanoparticles with an $88.3 \%$ photothermal conversion efficiency for photothermal therapy. Adv Mater. 2020;32:1907855.

31. Wu F, Chen L, Yue L, Wang K, Cheng K, Chen J, Luo X, Zhang T. Small-molecule porphyrin-based organic nanoparticles with remarkable photothermal conversion efficiency for in vivo photoacoustic imaging and photothermal therapy. ACS Appl Mat Interfaces. 2019; 11:21408-21416.

32. Jin CS, Lovell JF, Chen J, Zheng G. Ablation of hypoxic tumors with dose-equivalent photothermal, but not photodynamic, therapy using a nanostructured porphyrin assembly. ACS Nano. 2013;7:25412550.

33. Zou Q, Abbas M, Zhao L, Li S, Shen G, Yan X. Biological photothermal nanodots based on selfassembly of peptide-porphyrin conjugates for antitumor therapy. J Am Chem Soc. 2017;139:1921-1927. 
34. Li H, Yue L, Li L, Liu G, Zhang J, Luo X, Wu F. Triphenylamine-perylene diimide conjugate-based organic nanoparticles for photoacoustic imaging and cancer phototherapy. Colloids Surf B. 2021;205:111841.

35. Antaris AL, Chen H, Cheng K, Sun Y, Hong G, Qu C, Diao S, Deng Z, Hu X, Zhang B, et al. A smallmolecule dye for NIR-II imaging. Nat Mater. 2016;15:235-242.

36. Li S, Zhang W, Xing R, Yuan C, Xue H, Yan X. Supramolecular nanofibrils formed by coassembly of clinically approved drugs for tumor photothermal immunotherapy. Adv Mater. 2021;33:2100595.

37. Lei Z, Zhang F. Molecular engineering of NIR-II fluorophores for improved biomedical detection. Angew Chem Int Ed. 2021;60:16294-16308.

38. Wang Q, Dai Y, Xu J, Cai J, Niu X, Zhang L, Chen R, Shen Q, Huang W, Fan Q. All-in-one phototheranostics: single laser triggers NIR-II fluorescence/photoacoustic imaging guided photothermal/photodynamic/chemo combination therapy. Adv Funct Mater. 2019;29:1901480.

39. Kenry, Duan Y, Liu B. Recent advances of optical imaging in the second near-infrared window. Adv Mater. 2018;30:1802394.

40. Li S, Wang X, Hu R, Chen H, Li M, Wang J, Wang Y, Liu L, Lv F, Liang X-J, et al. Near-infrared (NIR)absorbing conjugated polymer dots as highly effective photothermal materials for in vivo cancer therapy. Chem Mater. 2016;28:8669-8675.

41. Huang L, Li Y, Du Y, Zhang Y, Wang X, Ding Y, Yang X, Meng F, Tu J, Luo L, et al. Mild photothermal therapy potentiates anti-PD-L1 treatment for immunologically cold tumors via an all-in-one and all-incontrol strategy. Nat Commun. 2019;10:4871.

42. Wang M, Rao J, Wang M, Li X, Liu K, Naylor MF, Nordquist RE, Chen WR, Zhou F. Cancer photoimmunotherapy: from bench to bedside. Theranostics. 2021;11:2218-2231.

\section{Figures}




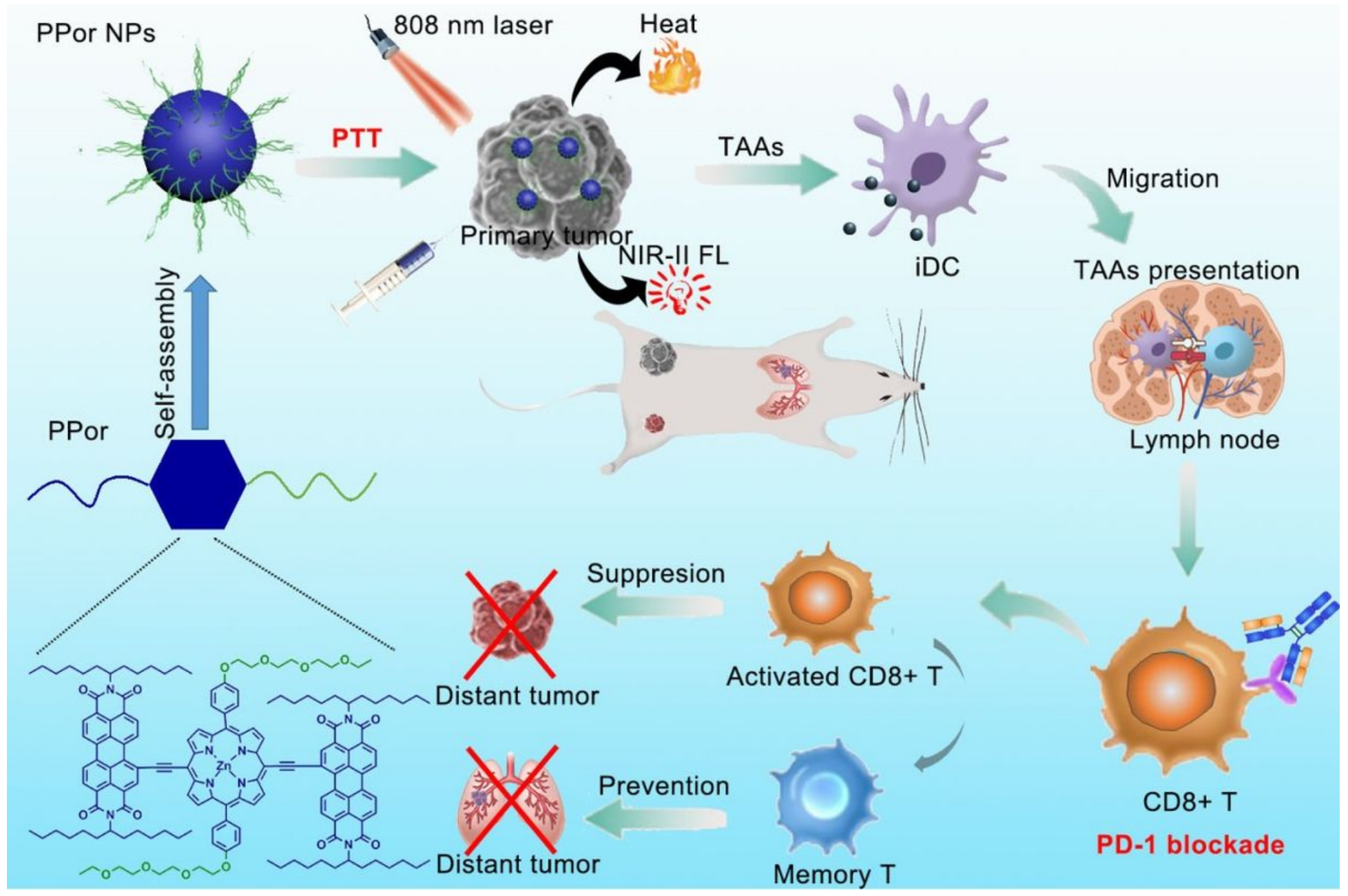

Figure 1

Schematic illustration of the formation and application of PPor NPs self-assembled from small molecules of porphyrin derivatives for NIR-II fluorescence imaging guided photothermal-immunotherapy. 
a
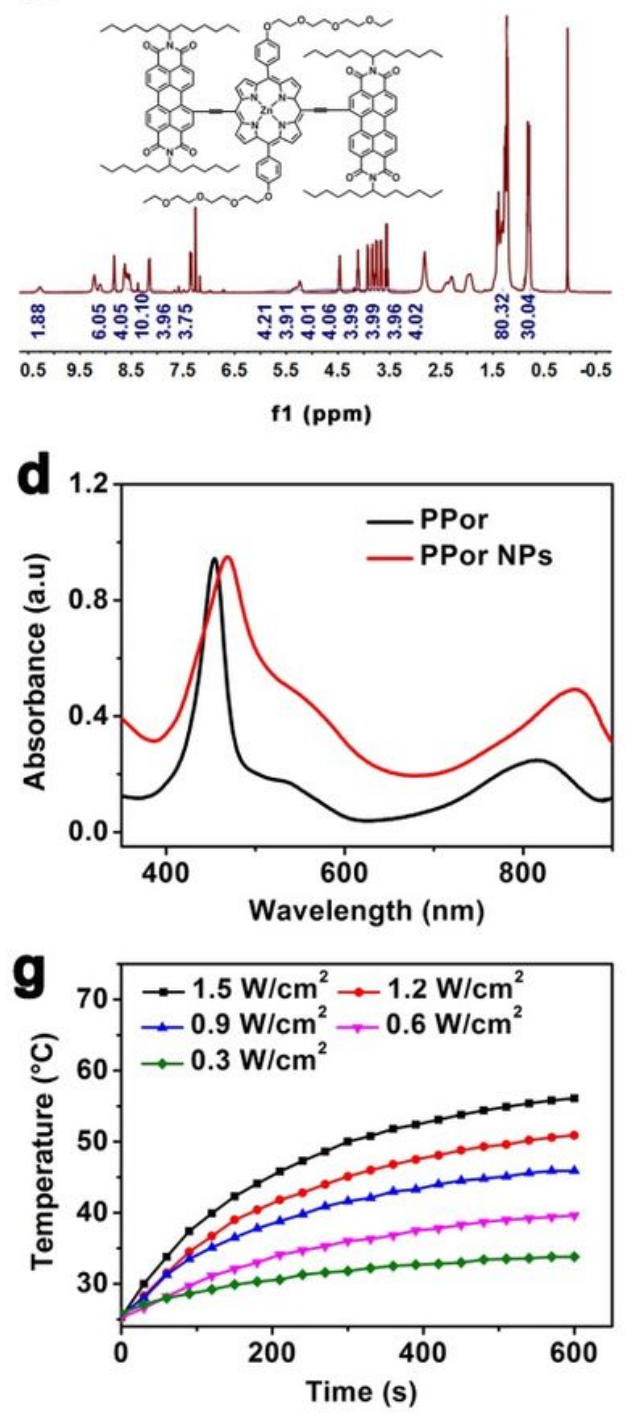
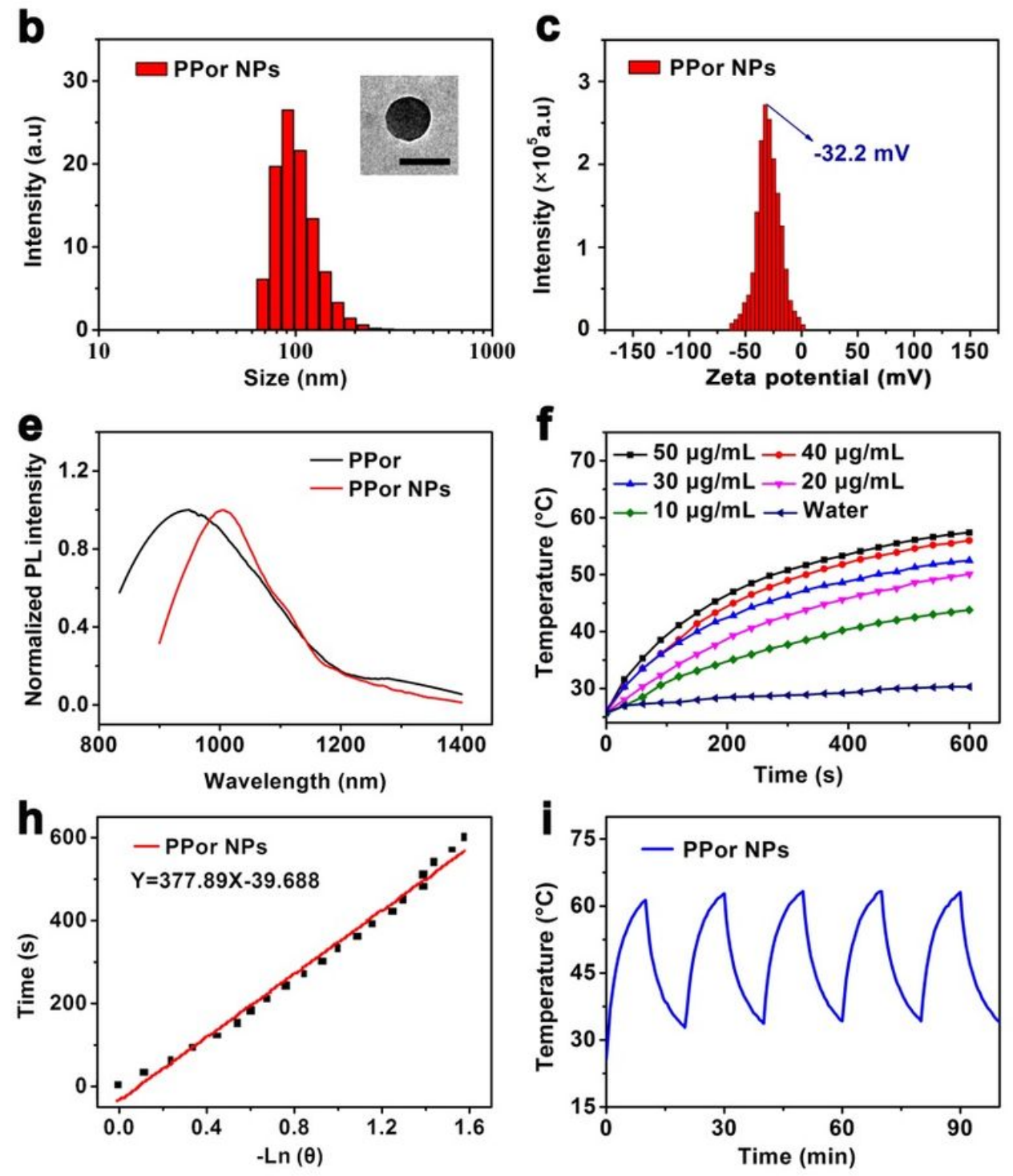

Figure 2

a Chemical structures and 1H NMR of the PPor. $b$ The size distribution of PPor NPs determined by DLS. The inset picture is the TEM image of the single PPor nanoparticle, Scale bar: $100 \mathrm{~nm}$. c Zeta distribution of PPor NPs determined by DLS. d UV-Vis-NIR absorbance of PPor and PPor NPs. e Fluorescence emission spectra of PPor in $\mathrm{CH} 2 \mathrm{Cl} 2$ and PPor NPs in water under $808 \mathrm{~nm}$ light excitation. $\mathrm{f}, \mathrm{g}$ Temperature curves of PPor NPs with different laser power densities (f) and different concentrations of PPor NPs (g) during $808 \mathrm{~nm}$ laser irradiation. h Plot of cooling time versus - In ( $\theta$ ). i Photothermal stability of PPor NPs upon five "On-to-Off" laser irradiation. 

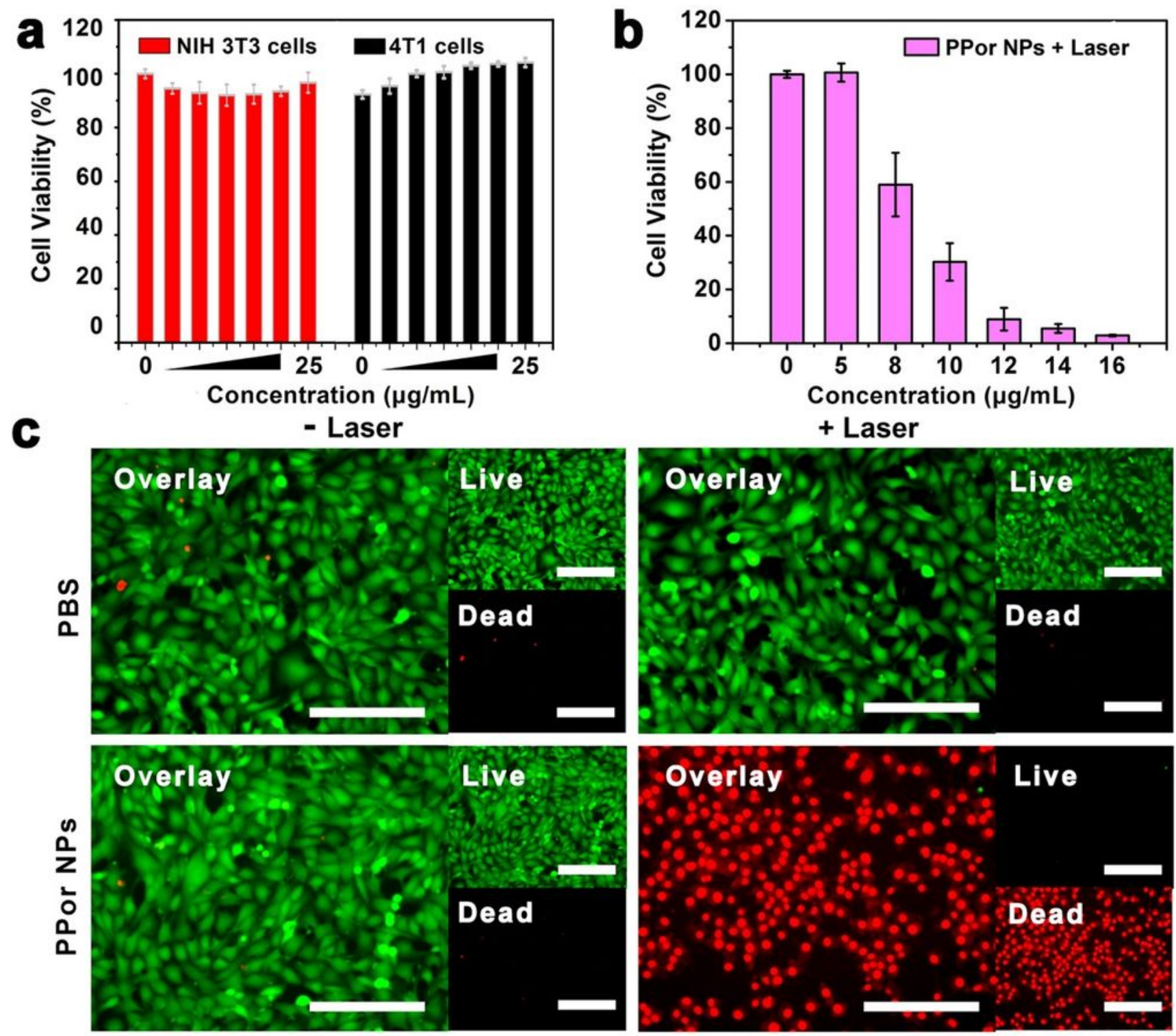

0
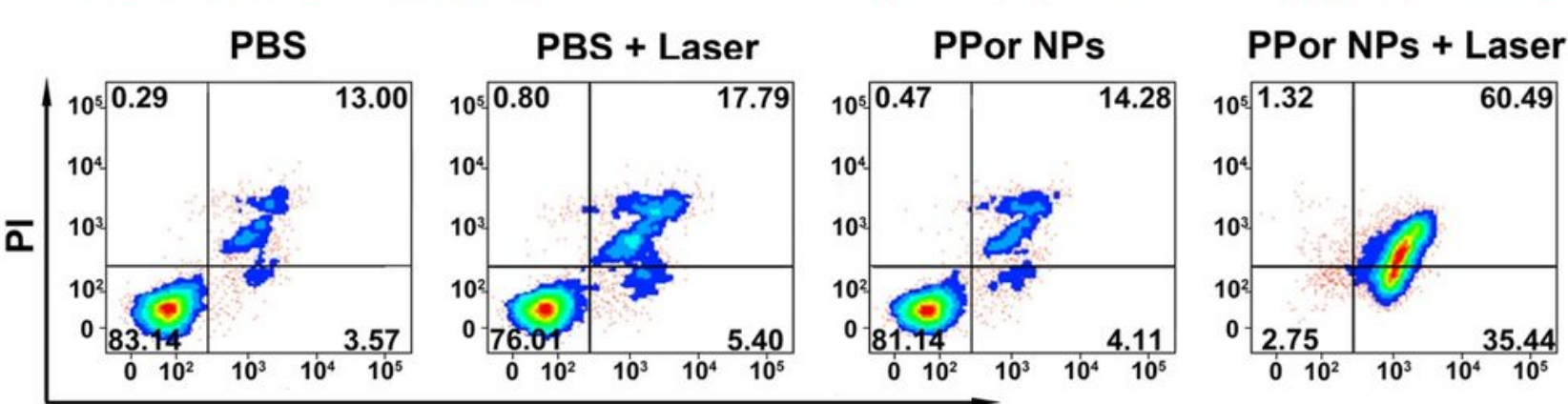

Annexin-FITC

Figure 3

a Cell viability of NIH $3 \mathrm{~T} 3$ cells and $4 \mathrm{~T} 1$ cells treated with PPor NPs at various concentrations without $808 \mathrm{~nm}$ laser irradiation $(n=4)$. b Cell viability of $4 T 1$ cells treated with PPor NPs at various concentrations with $808 \mathrm{~nm}$ laser irradiation $(0.8 \mathrm{~W} / \mathrm{cm} 2)$ for $10 \mathrm{~min}(\mathrm{n}=4)$. c Live/dead assay of 4T1 cells treated with PBS, PBS + Laser, PPor NPs, or PPor NPs + Laser, as observed by fluorescence 
microscope. Scale bar: $100 \mu \mathrm{m}$. d Cell apoptosis of 4T1 cells treated with PBS, PBS + Laser, PPor NPs, or PPor NPs + Laser, which was examined by FACS.
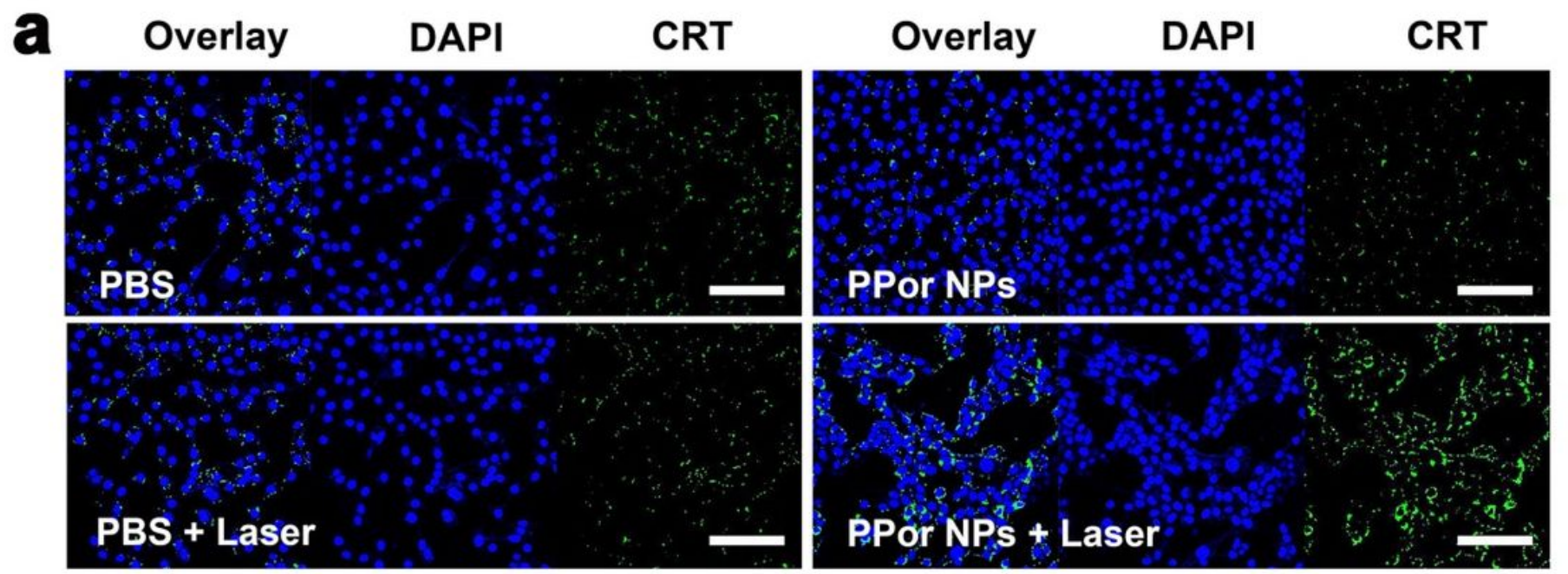
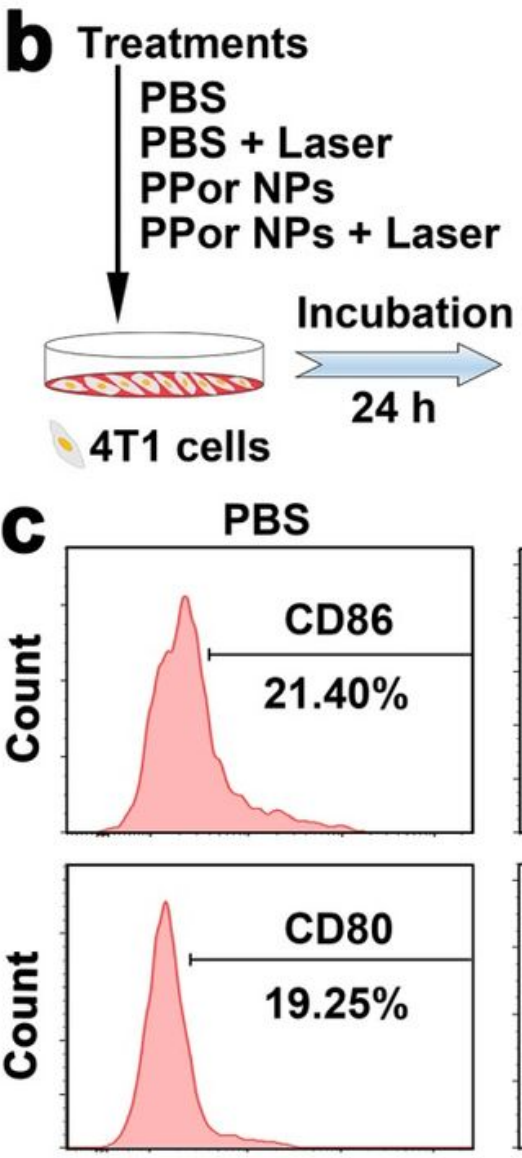

Analysis of DC maturation
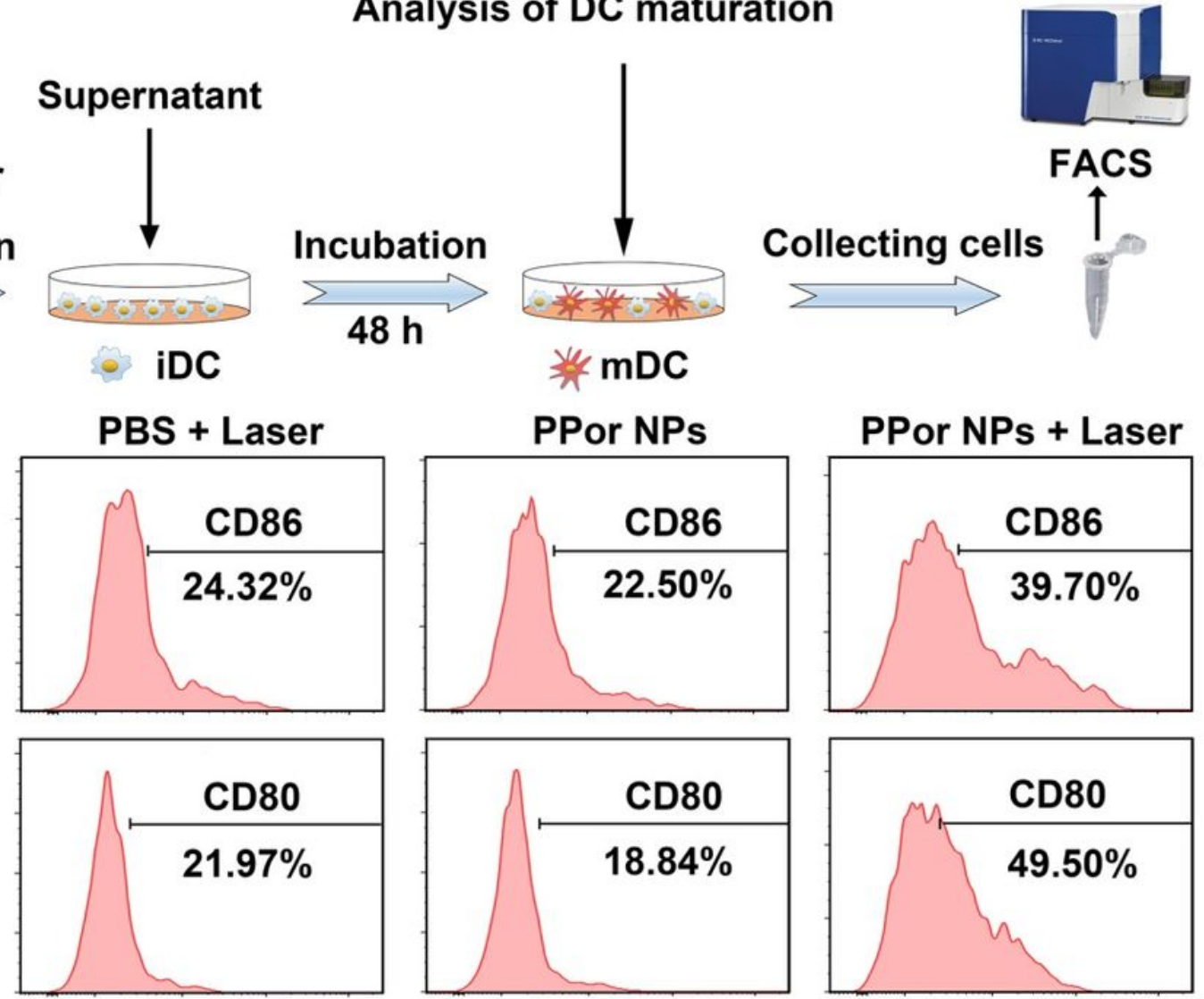

Figure 4

a Immunofluorescence of CRT exposure on 4T1 cells treated with PBS, PBS + Laser, PPor, or PPor + Laser. Scale bar: $100 \mu \mathrm{m}$. b Schematic illustration of the experimental design of DC maturation in vitro. C The frequency of BMDCs maturation induced by $4 \mathrm{~T} 1$ cells with different treatments as indicated. 


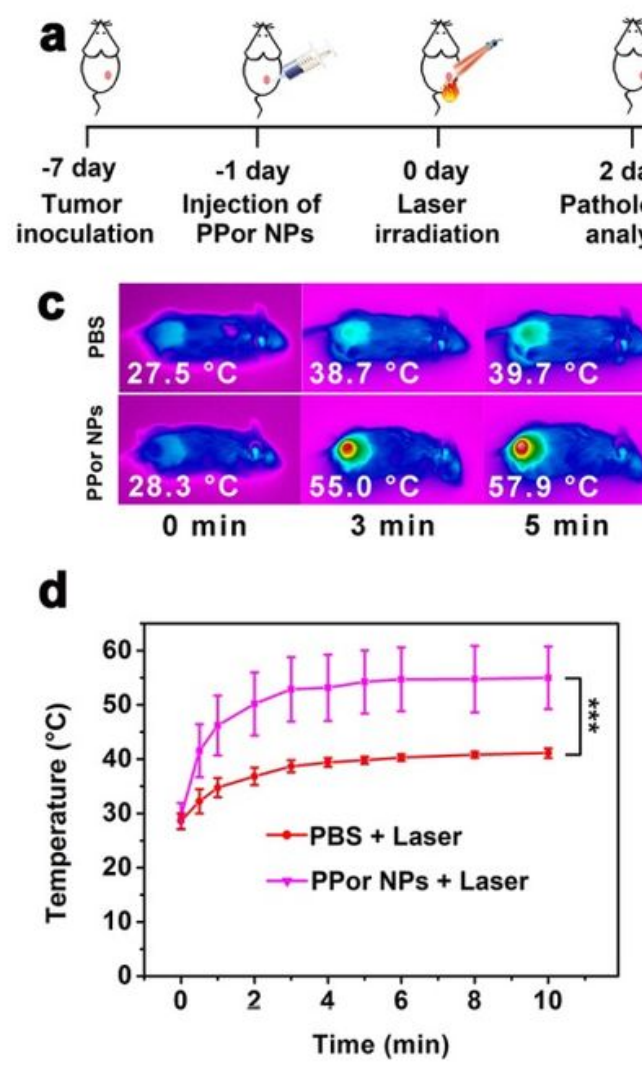

g

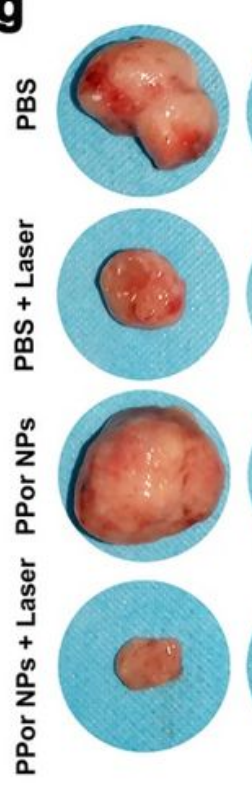

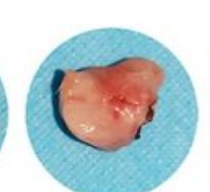
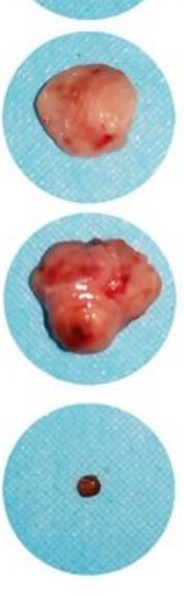
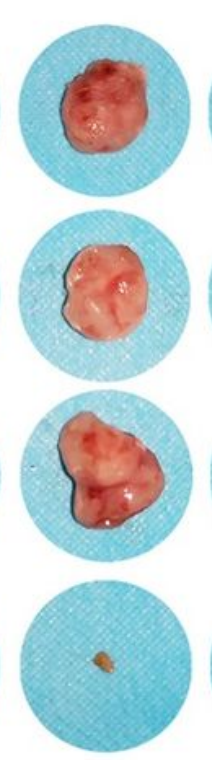

e

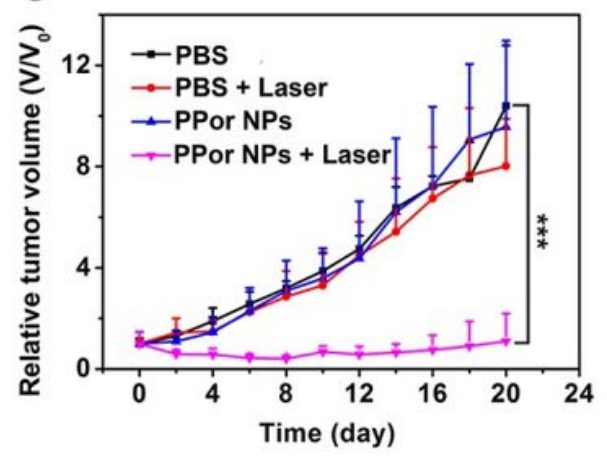

h
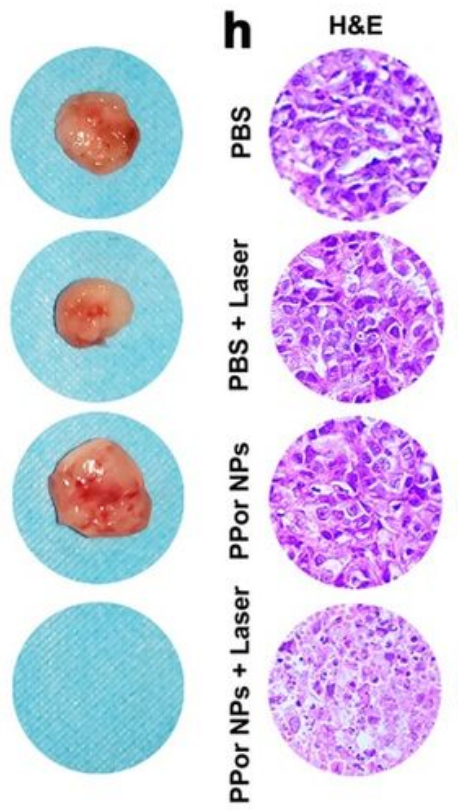
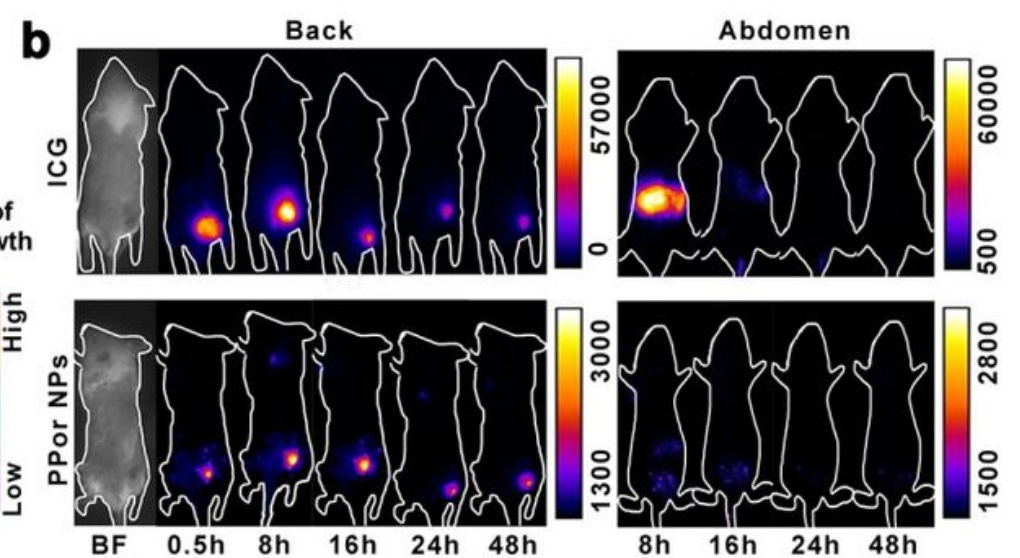

f
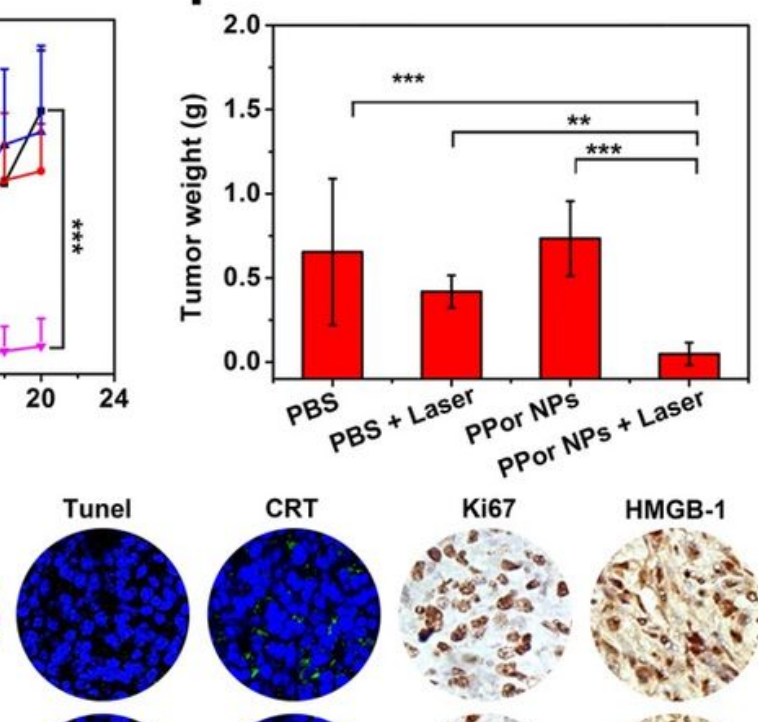

HMGB-1
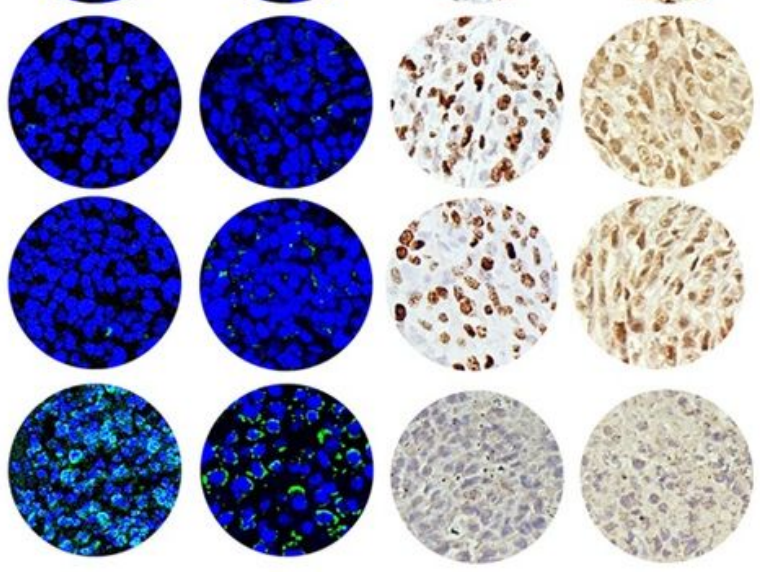

\section{Figure 5}

a Schematic illustration of treatment schedule for PPor NPs-mediated PTT effect in mice. b In vivo NIR-II fluorescence imaging of the mouse bearing 4T1 tumor at different time points after intratumoral injection. c, d IR thermal images of $4 \mathrm{~T} 1$ tumor-bearing BALB/c mice (c) and temperature curve of tumor (d) under the $808 \mathrm{~nm}$ irradiation $(0.8 \mathrm{~W} / \mathrm{cm} 2)$. e-g Relative tumor volume curves (e), average tumor weights $(\mathrm{f})$ and tumor photograph $(\mathrm{g})$ of the mice received different treatments. ${ }^{*} \mathrm{P}<0.05,{ }^{\star \star *} \mathrm{P}<0.01$, $* \star * P<0.001 ; n=4$ per group). $\mathrm{h}$ H\&E staining, Ki67 antigen immunohistochemistry staining, Tunel 
staining, CRT immunofluorescence staining, as well as HMGB-1 immunohistochemical staining images of tumor sections after different treatments.
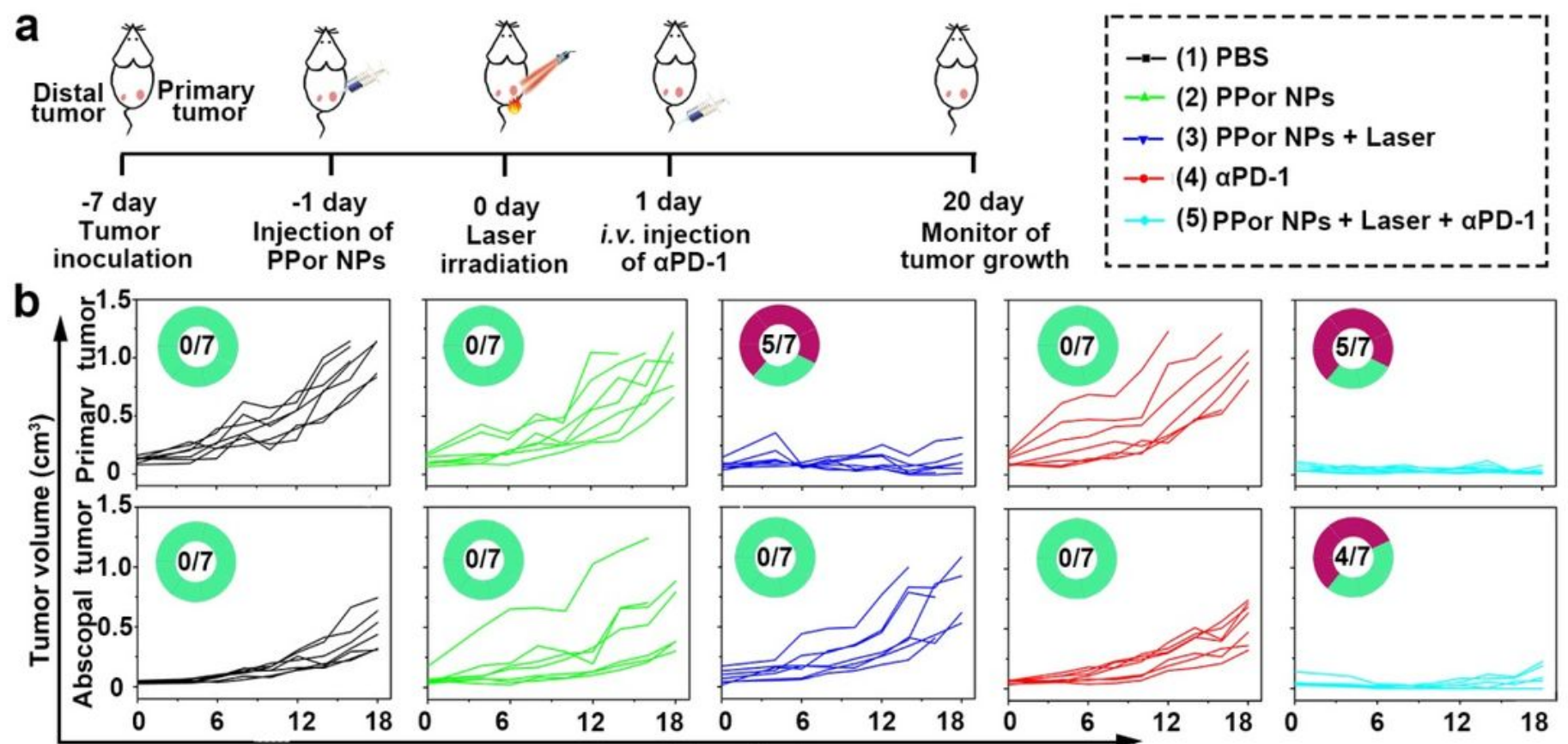

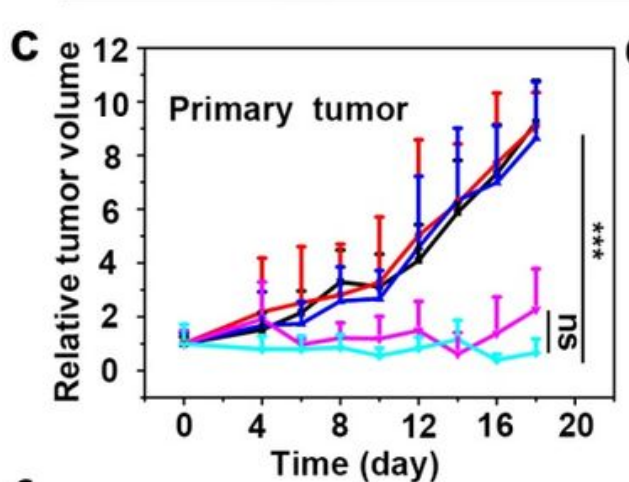

f

(1)

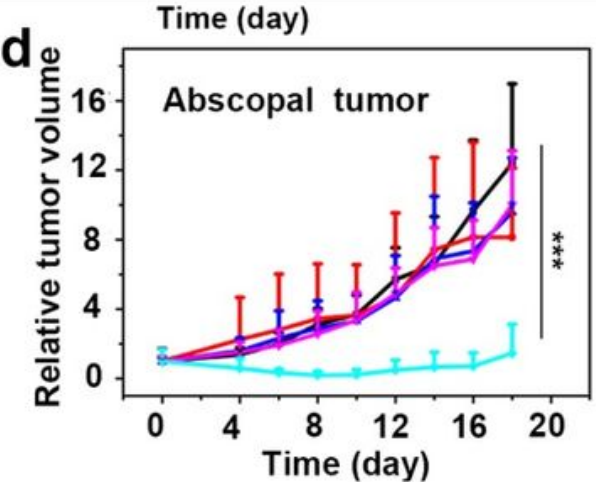

(3)

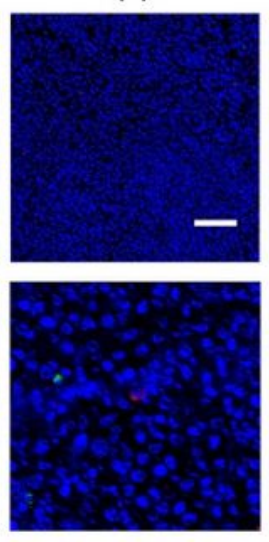

(4)
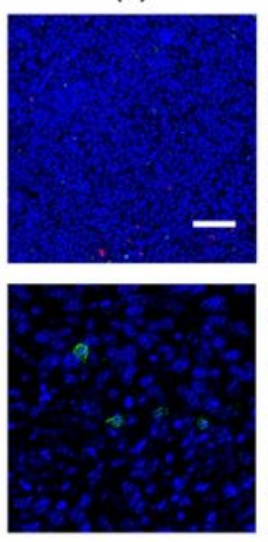

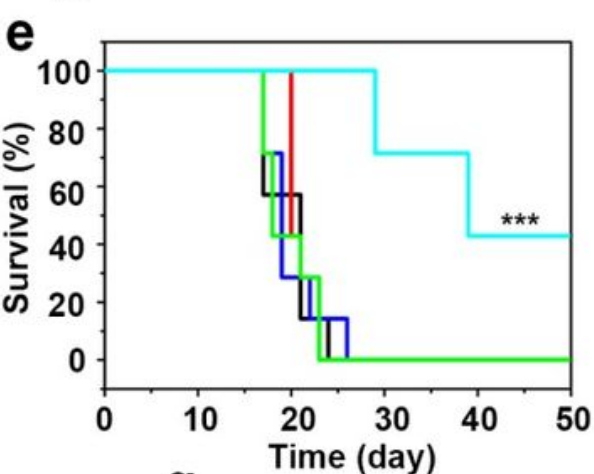

(5)

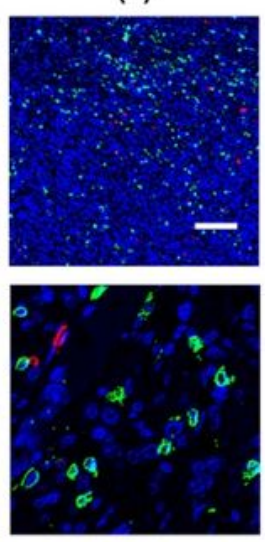

g

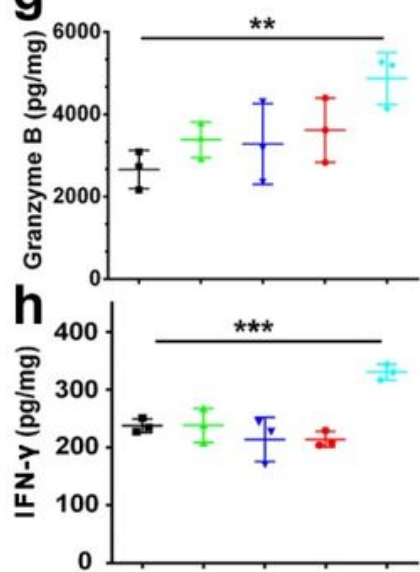

Figure 6

a Schematic illustration of treatment schedule of photothermal therapy combined with immunotherapy for primary tumor and abscopal tumor. b Tumor growth curves of the primary tumors and abscopal tumors after various treatments (PBS, PBS+Laser, PPor NPs, PPor NPs+Laser). c, d Relative tumor curves 
of primary tumor (c) and abscopal tumor (d) by the above treatments $\left({ }^{\star} \mathrm{p}<0.05,{ }^{\star \star} \mathrm{p}<0.01,{ }^{\star \star \star} \mathrm{p}<0.001\right.$; $n=7$ ). e Survival curves of mice by the above treatments ( $\left.{ }^{*} p<0.05,{ }^{\star \star} p<0.01,{ }^{\star \star *} p<0.001 ; n=7\right) . f$ Immunofluorescence staining of the tumor sections (CD4+ T cells-red and CD8+ T cells-green) of the abscopal tumor with different enlargement factors. Scale bar: $100 \mu \mathrm{m} . \mathrm{g}$, h The ELISA analysis of cytokine levels (IFN- $y$, Granzyme B) in abscopal tumor ( ${ }^{*} p<0.05,{ }^{\star \star} p<0.01,{ }^{\star \star *} p<0.001 ; n=3$ ).

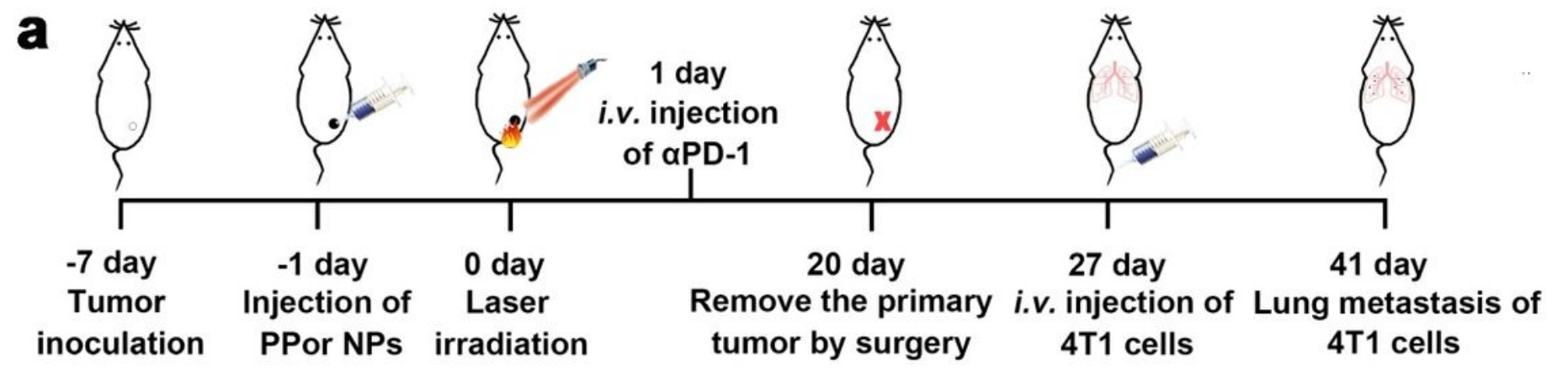

b

b PBS

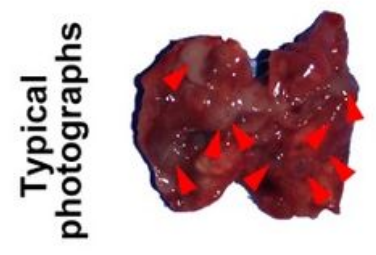

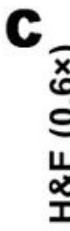

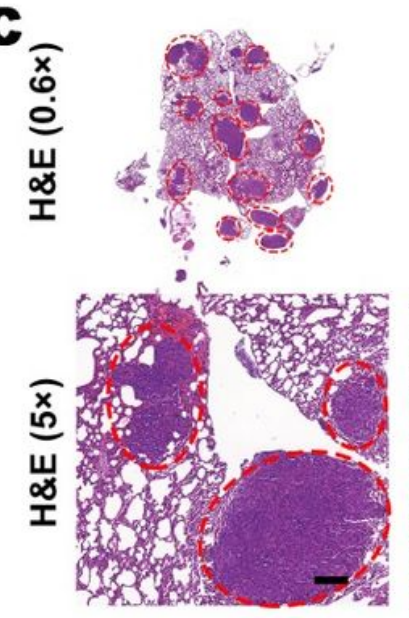

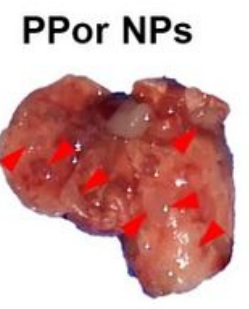

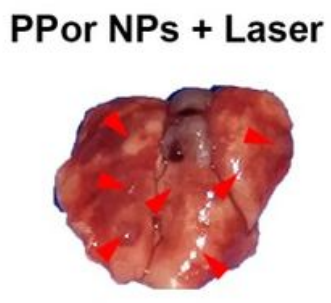

aPD-1

PPor NPs + Laser + $\alpha$ PD-1
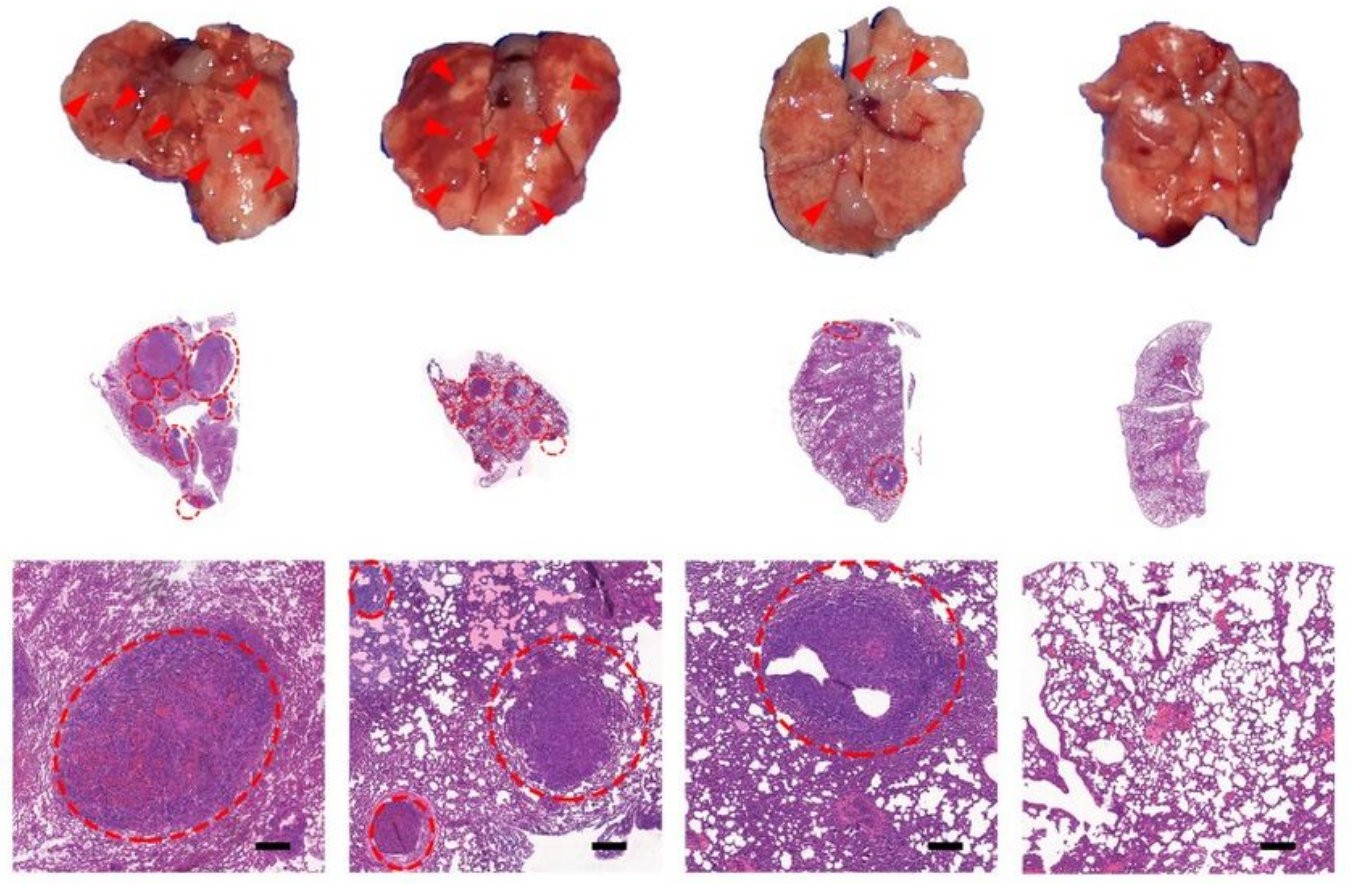

Figure 7

a Schematic illustration of the course of combination therapy that can inhibit the metastasis of BALB/c mice. b, c Representative photographs (b) and H\&E staining (c) of lungs in each group.

\section{Supplementary Files}

This is a list of supplementary files associated with this preprint. Click to download.

- SupplementaryInformation.docx 